\title{
Landslide hazard zones determination on open pit mine edge by circular seismic sounding method
}

\author{
Mikhail Karablin ${ }^{1 .}$, Dmitry Sirota ${ }^{2}$, Sergey Prostov ${ }^{2}$, and Olmos Abdurasulov ${ }^{3}$ \\ ${ }^{1}$ «Kuzbassgiproshakht» OJSC, Technical Department, 650000 Kemerovo, the Russian Federation \\ ${ }^{2}$ T.F. Gorbachev Kuzbass State Technical University, Mining Institute, 650000 Kemerovo, the \\ Russian Federation \\ ${ }^{3}$ «Uzkumirsanoat ITLI» LTD, Tashkent, The Republic of Uzbekistan
}

\begin{abstract}
Herein there is an algorithm and method of processing the experimental data obtained by a circular seismic sounding method that makes it possible to increase accuracy in determining an anisotropy coefficient of the ground mechanical properties. The coefficient value is mutually related to the deformation processes at the initial stages of landslide formation. There are results of data processing of the field seismic studies of the adjacent ground at the Angrenskiy coal mine that allow establishing boundaries for the potentially landslide-prone zones, as well as a numerical criterion to predict rock stability under the given conditions.
\end{abstract}

\section{Introduction}

To improve evaluation stability accuracy for the various type of slope structures (open pits side, dams, dumps, embankments, and excavations etc.), physical and mechanical property details of the studied ground are required. In this regard, traditional methods of engineering and geological surveys and hydrogeological monitoring are supplemented by geophysical measurements. Herewith, well geophysical logging, ultrasound, seismic, nuclear-physical and electrical methods provide the required meticulous database, however are associated with great drilling operations $[1,2]$. Wells-free geophysical methods, as much less laborious, have got priority development, and methods of sounding from the earth's surface (from underground workings) are also widely used. Currently, geo-acoustics and geo-radar location methods are being developed most actively. Improvement of geophysical methods goes in two directions: expanding of measuring equipment capabilities; updating of software and methodological support at processing and interpretation of experimental databases [3].

Significant success was achieved in solving a problem of geophysical monitoring using underground geo-technology. One of the most successful projects is an active implementa-

*Corresponding author: karablin.mm@gmail.com 
tion of the ground acoustic monitoring system (GAMS) and forecast of dynamic phenomena at the SUEK-Kuzbass mines. Source of the acoustic signal in the system is an effect caused by mining equipment on face or development working. Acoustic signal processing is made continuously throughout the entire coal extraction cycle, and predictive parameters are computed based on the resulting average spectrum of the acoustic signal [4].

The functionality of the GAM system is as follows [5-7]: forecast of the potentially hazardous areas on coal and gas emissions, rock-bursts, dynamic rock failure in workings with intense gas release in presence of a gas-bearing satellite bed, abrupt coal extrusion; prediction of geological disturbances that are dangerous for dynamic phenomena ahead of a moving face; operational management of the coal seam hydraulic loosening process; monitoring of safety and efficiency of the pilot relieve holes; VAT definition for the face parameters; notification regarding deterioration of dynamic phenomena state in the face and high rock pressure.

Seismic monitoring methods applying various measurement design, techniques and software to interpret their results were used to evaluate stability of the adjacent ground for open pit mines [8], physical and mechanical properties of soils [9], to determine ground failure and observe karst occurrence $[10,11]$, to evaluate rock strength and determine anomalous tectonic zones [12-14], to clarify conditions of formation and gas content [1517], to control stress-strain state of sand-clay soil and hard rocks [18, 19].

Studying state and properties of the adjacent coal mass, seismic sounding method is very perspective that allows covering large areas of potentially landslide-hazardous ground.

\section{Research methods}

Seismic surveys of the adjacent ground in the Angrenskiy open pit coal mine were carried out by the expedition of JSC Ugol, Republic of Uzbekistan.

A circular seismic sounding method has been used, the essence of which could be presented as follows. The seismic station included a hammer point located in the center of a circle with a radius of $10 \mathrm{~m}$ and 12 geophones fixed at specially prepared points along a circle with an angular step $\varphi=30^{\circ}$. Exitation was excited by 10-20 thumps on the mass surface by a tamper; registration was carried out by the Lakkolit 24-M seismic station in the accumulation mode using geophones 9C-20dx.

Main result of the seismic sounding data processing is an anisotropy coefficient $\vec{K}_{a}$, the value is determined by the ratio:

$$
K_{a}=\frac{V_{p_{\mathrm{II}}}}{V_{p_{\perp}}},
$$

where $V_{p_{\text {II }}}, V_{p_{\perp}}-$ P-wave velocity in directions, respectively, parallel to the plane of soil softening, and perpendicular to it.

The $K_{a}$ value totally characterizes a potential risk of landslide formation in the studied area, the severity of which is defined by strength and deformation decrease in the ground properties and deformation development. Magnitude and direction of the $\vec{K}_{a}$ vector can be determined from circle charts of the time intervals of the longitudinal wave $t_{i}$ arrival from the angular coordinate, a characteristic view is shown in Fig 1. 

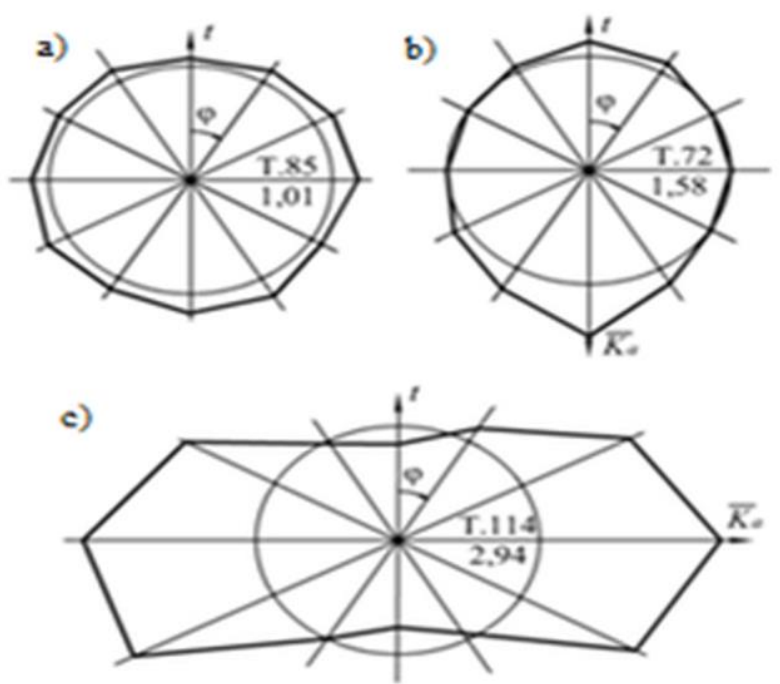

Fig. 1. Characteristic circle charts of $t_{i}(\varphi)$ time arrival of the longitudinal wave $K_{a}=V_{p \max } / V_{p \min }=1737 / 1706=1.01$ (a); $K_{a}=2449 / 1540=1.58$ (b); $K_{a}=2729 / 927=2.94$ (c)

To improve accuracy of definition of the $\vec{K}_{a}$ vector parameters it is necessary to develop a method for ground approximation of experimental data using an ellipse equation. One of the effective methods for geophysical data processing is discussed below.

Assume that there are pairs of values $V_{k}$ and $\varphi_{k}$, where $\varphi$ - azimuth angle, and $V-$ acoustic wave velocity (polar radius). The broken curve chart $V_{k}=V\left(\varphi_{k}\right)$ in the polar coordinate system can be approximated by rotated, relatively to the standard coordinate system, some angle $\gamma$ by ellipse with semi-axes $a, b$.

It is required to determine values of the semi-axes $a, b$ and angle $\gamma$. For this we are to use the least squares method, which is reduced to finding minimum of three variables function $a, b, \gamma$ of the following type:

$$
F=\sum_{k}\left[V_{k}-V\left(a, b, \gamma, \varphi_{k}\right)\right]^{2},
$$

where $V(a, b, \gamma, \varphi)$ - ellipse theoretical equation in the polar coordinate system $O V \varphi$.

Relatively to the rotated coordinate system, the canonical equation of an ellipse with a center at the beginning of the coordinate system has a form:

$$
\frac{V_{x}^{2}}{a^{2}}+\frac{V_{y}^{2}}{b^{2}}=1 \text {. }
$$

Relationship between the original and rotated on angled $\gamma$ coordinate systems is given by the matrix expression of rotation:

$$
\left(\begin{array}{c}
V_{X} \\
V_{Y}
\end{array}\right)=\left(\begin{array}{cc}
\cos \gamma & \sin \gamma \\
-\sin \gamma & \cos \gamma
\end{array}\right) \cdot\left(\begin{array}{c}
V_{x} \\
V_{y}
\end{array}\right) .
$$

Coordinates $V_{x}, V_{y}$ in the polar coordinate system are defined by expressions: 


$$
\begin{aligned}
& V_{x}=V \cos \varphi, \\
& V_{y}=V \sin \varphi .
\end{aligned}
$$

Substituting (6) and (5) in (4), and then in (3), we obtain an expression relatively to velocity $V=V(\varphi)$ of the following form:

$$
V=\frac{a \cdot b}{\left[A^{2} a^{2}+B^{2} b^{2}\right]^{1 / 2}},
$$

where $A=\cos \varphi \cos \gamma+\sin \varphi \sin \gamma, B=\sin \varphi \cos \gamma-\cos \varphi \sin \gamma$.

Since due to the use of functions $\sin \gamma$ and $\cos \gamma$ in (7) the obtained function (2) is multimodal, then the use of any gradient improving algorithms to search for the minimum of function (2) is inefficient. In this situation a possible way out is to use optimization methods of random search that allow detecting not only local minima, but also global ones. One of the simple and at the same time effective method is the 'simulated annealing' method, which is an option of the classical statistical test method (Monte Carlo). The calculation flow chart is shown in Fig. 2.

The algorithm includes the following steps.

Step 1. Set starting initial values of variables $a_{0}=1 ; b_{0}=1 ; \gamma_{0}=0 ; T_{0}=5$; calculate function value (2) in the point $F_{\text {start }}=F\left(a_{0}, b_{0}, \gamma_{0}\right)$; set the start value of the annealing parameter $T_{0}=5$ and its lower limits $T_{\min }=0,005$. Steps $2-5$ repeated until the condition is met $T_{0}>T_{\min }$.

Step 2. Set new values $a_{\text {new }} ; b_{\text {new }} ; \gamma_{\text {new }}$; calculate function value (2) in this point $F_{\text {new }}=F\left(a_{\text {new }} ; b_{\text {new }} ; \gamma_{\text {new }}\right) ;$ calculate the difference $\Delta=F_{\text {new }}-F_{0}$.

Step 3. If the condition $\Delta<0$ is met, then update the starting values, reduce parameter $T_{0}$ and go to step 2; if the condition is not met, go to step 4.

Step 4. Generate random number $p \in[0 ; 1]$, calculate the probability of updating of the starting value as per the formula:

$$
P=\exp \left(-\Delta / T_{0}\right)
$$

Step 5. If the condition $P>p$ is met, then update the starting values, reduce parameter $T_{0}$ and go to step 2; if the condition is not met, then simply go to step 2. 


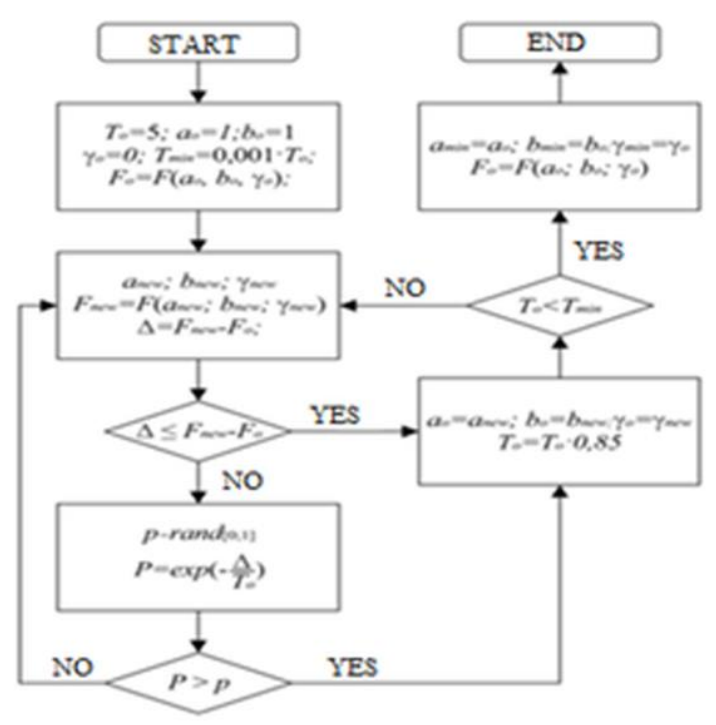

Fig. 2. Calculation flowchart

The application of this algorithm is to be considered in the following test example, which will consist of several stages.

1. Generation of initial data in order to replace experimental data.

Assume that the ellipse parameters take values $a=5, b=2, \gamma=45^{\circ}$. Let's form a uniform grid based on the polar coordinate $\varphi_{k} \in[0 ; 2 \pi]$ with step $h_{\varphi}=\frac{\pi}{18}$. To prevent an 'inverse crime' effect we will shift each node of this grid on a random amount within the half-step of the grid in a way to get an uneven grid.

In each node of the new grid, we calculate the value of the polar radius by the formula (7): $V_{k}=V\left(a, b, \gamma, \varphi_{k}\right)$. We change each value of the polar radius on a random value using the following formula $V^{R}=V \cdot\left[1+\frac{\text { rand }}{2}\right]$, where rand - random number, evenly distributed on the segment $[-1 ; 1]$.

2. Definition of theoretical values of the ellipse parameters that is close to the original one. Set starting initial parameters $a=1, b=1, \gamma=0^{o}$ - i.e. unit circle parameters. Applying formula (1) and the mentioned 'simulated annealing' method hereinabove, we select the ellipse parameters. Changing parameters $a, b, \gamma$, it is necessary to consider the positivity of the half axes $a, b$, as well as the angle change interval $\gamma \in\left[0^{\circ} ; 90^{\circ}\right]$. As a result, we get the following values: $a=4.813, b=2.087, \gamma=44.996^{\circ}$, wherein the value $\mathrm{F}$ from formula (2) is decreased from 170 at starting values of parameters up to 24 at the obtained optimal ones. Total calculation time was 10 minutes. Experimental and theoretical data are in Fig. 3. 


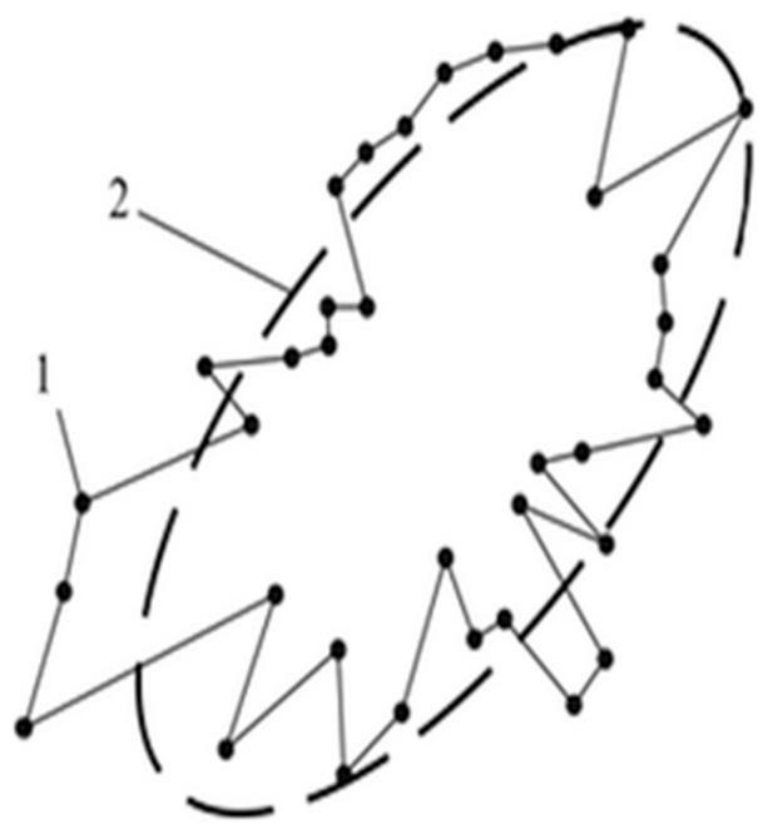

Fig. 3. Processing results: 1-experimental data; 2-theoretical dependence

If the angle of the ellipse is known beforehand, the speed of finding the optimal solution increases and the total time is 1 minute. In this case, the values of the found half axes will be equal to: $a=4.901, b=2.035$, and value $F=40.823$.

\section{Results and discussions}

The pilot site diagram on the southern side of the Angrenskiy coal mine (Republic of Uzbekistan) in the area of the emerging landslide is shown in Fig. 4. The pilot site included 11 seismic stations located on an area of over 100 hectares. The total length of the geophysical profile was $1.0 \mathrm{~km}$, and the distance between neighboring seismic stations was from 50 to $100 \mathrm{~m}$.

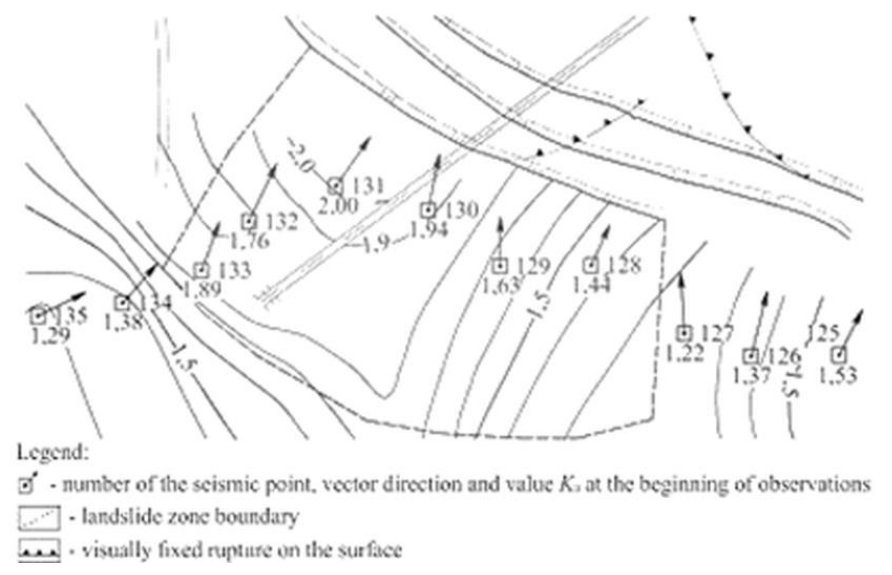

Fig. 4. Pilot site diagram on the southern side of the coal mine 
Processing and analysis of the field measurement data was carried out as per the method hereinabove.

Table 1. Results of determining the anisotropy coefficient $K_{a}$

\begin{tabular}{|l|c|c|c|c|c|c|c|c|c|c|c|}
\hline \multirow{2}{*}{$\begin{array}{l}\text { Observa- } \\
\text { tion point }\end{array}$} & \multicolumn{10}{|c|}{ Number of the seismic point for circular seismic sounding method (CSSM) } \\
\cline { 2 - 12 } & $\mathbf{1 3 5}$ & $\mathbf{1 3 4}$ & $\mathbf{1 3 3}$ & $\mathbf{1 3 2}$ & $\mathbf{1 3 1}$ & $\mathbf{1 3 0}$ & $\mathbf{1 2 9}$ & $\mathbf{1 2 8}$ & $\mathbf{1 2 7}$ & $\mathbf{1 2 6}$ & $\mathbf{1 2 5}$ \\
\hline Start & 1.29 & 1.38 & 1.89 & 1.76 & 2.00 & 1.94 & 1.63 & 1.44 & 1.22 & 1.37 & 1.53 \\
\hline 2 months & & 1.38 & 2.28 & 1.71 & 2.68 & 2.13 & 1.30 & 2.13 & 1.53 & 1.48 & 1.56 \\
\hline 12 months & 1.73 & 1.00 & 2.25 & 1.71 & 2.80 & 2.13 & 1.45 & 1.66 & 1.55 & 1.48 & 1.66 \\
\hline 15 months & 1.73 & 1.00 & 1.50 & 1.76 & 2.36 & 2.25 & 1.67 & 1.45 & 1.48 & 1.32 & 1.15 \\
\hline 25 months & & 1.39 & 2.26 & 1.71 & 2.53 & 2.13 & 1.48 & 1.95 & 1.35 & 1.32 & 1.35 \\
\hline 33 months & 1.58 & & 1.55 & 1.29 & 1.95 & 1.71 & 1.77 & 1.53 & 1.22 & 1.04 & 1.06 \\
\hline 36 months & 1.38 & 1.26 & 1.05 & 1.29 & 1.60 & 1.27 & 1.47 & 1.53 & 1.15 & 1.04 & 1.06 \\
\hline
\end{tabular}

The Fig. 5 presents graph of variants of the value $K_{a}$ on seismic points (SP), located approximately parallel to the open-pit edge, depending on the time: near the edge (SP 131), in the middle (SP 132) and rear (SP 134) parts of the potentially landslide-prone zone.

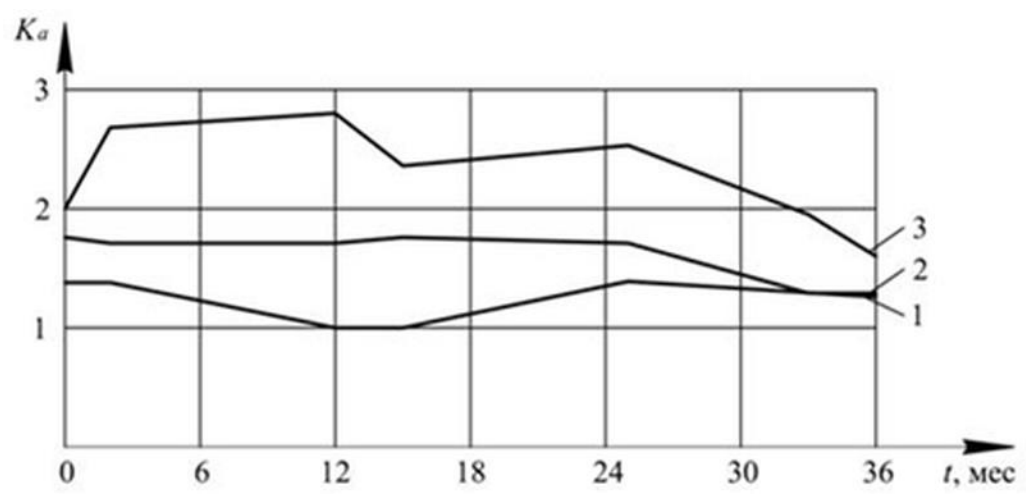

Fig. 5. Graph of variants of the value Ka depending on the time of observation: $1-t=0 ; 2-t=15$ months; $3-t=25$ months; $4-t=36$ months; $1-$ SP 134; $2-$ SP 132; $3-$ SP 131

From the obtained results, it follows that at the boundaries of the geophysical profile, the $K_{a}$ value is in the range $K_{a}=1.04-1.73$, while in its central part it reaches the maximum values $K_{a}=2.00-2.80$ on the interval $t=12-24$ months. Later on, with the formation of ruptures on the surface of the open-pit edge (see Fig.4) ground is unloaded that leads to decrease in the anisotropy of the ground mechanical properties and a corresponding decrease in the parameter $K_{a}$ below the limit $K_{a}<1.6$.

\section{Conclusions}

The criterion of the potential landslide hazard based on the results of circular seismic sounding was experimentally established: $K_{a}>1.6$. Using the criterion, the boundaries in the plan of the landslide zone are set (see Fig.4), the approximate dimensions of which were $500-300 \mathrm{M}$. 


\section{References}

1. V. L. Shkuratnik, P. V. Nikolenko, A. A. Kormnov, Physical and technical problems of mineral development, 3, 41-47 (2015)

2. V.V. Popov, H.A. Ershov, Geophysics, 2, 57-63 (2011)

3. A. P. Egorov, V. A. Ryzhov, Coal, 10, 1123, 29 (2019)

4. K. N. Kopylov, O. V. Smirnov, A. I. Kulik, Mine information and analytical Bulletin, 7, $82(2015)$

5. K. N. Kopylov, O. V. Smirnov, A. I. Kulik, P. V. Potapov, Coal, 7, 44 (2015)

6. O. V. Smirnov, A. I. Kulik, Mine information and analytical Bulletin, 5, 352 (2017)

7. O. V. Smirnov, A. I. Kulik, E. A. Lapin, Coal, 11, 76 (2015)

8. O. G. Besimbayeva, E. N. Khmyrova, E. A. Oleynikova, Interexpo Geo-Siberia, 146$150(2016)$

9. E. V. Denisova, A. I. Konurin, GIAB, 8, $176(2014$

10. T. V. Baibakova, GIAB, 12, 236 (2009)

11. D. A.Malovichko, O. I.Kadebskaya, D. Yu.Shulakov, P. G.Butyrin, Earth Physics, 1, $62(2010)$

12. I. A.Sapfirov, A.M.Mukhametshin, A. B.Chugaev, GIAB, 12, 307 (2010)

13. O. Tailakov, S. Sokolov, M. Makeev, A. Kormin, E3S Web of Conferences, Electronic edition (2018)

14. O.V. Taylakov, S.V. Sokolov, A.V. Gerasimov, A.A. Kolmakova , GIAB, 49, 275 (2018)

15. O.V. Taylakov, V.I. Ovchinnikov, S.V. Sokolov, Geotechnical Mekhanika, 88, 22 (2010)

16. E.A. Novikov, V.L. Shkuratnik, R.O. Oshkin, M.G. Zaitsev, Soil Mechanics and Foundation Engineering, 54, 2, 81 (2017)

17. P.V. Nikolenko, V.L. Shkuratnik, Journal of Mining Science, 50, 6, 1088 (2014)

18. L.S. Zagorskiy, V.L. Shkuratnik, Acoustical Physics, 60, 3, 297 (2014)

19. L.S. Zagorskiy, V.L. Shkuratnik, Acoustical Physics, 59, 2, 197 (2013) 